Background and aims $\gamma \delta \mathrm{T}$ cells are important in combating infectious agents and tumour cells. Their role in the pathogenesis of rheumatoid arthritis (RA) remains unknown.

Methods 68 patients with rheumatoid arthritis, 21 patients with osteoarthritis and 21 healthy controls were enrolled in the study. Peripheral V $\delta 2 \mathrm{~T}$ population, apoptosis, proliferation, chemokine receptor expression and pro-inflammatory cytokine secretion were quantified by flow cytometry. The infiltration of V82 T cells within synovium was examined by immunohistochemistry and flow cytometry. The effect of TNF- $\alpha$ and IL6 on V $82 \mathrm{~T}$ migration was determined by flow cytometry and trans-well migration assay.

Results The percentage of peripheral V $\delta 2 \mathrm{~T}$ cells of active RA were significantly decreased compared with healthy controls, which were negatively correlated with the disease activity indexes including DAS28, CRP and ESR. However, the V82T cells infiltrated in the synovium of RA were increased compared with OA $(\mathrm{p}<0.05)$. Comparing with OA V $22 \mathrm{~T}$ cells, both peripheral and synovium V $82 \mathrm{~T}$ cells of RA produced higher level of IFN- $\gamma$ and IL-17 $(\mathrm{p}<0.05)$. The chemokine receptor CCR5 and CXCR3 expressed on V82T cells in RA were significantly higher than $\mathrm{HC}$ and $\mathrm{OA}$ patients $(\mathrm{p}<0.05)$, which were induced by TNF- $\alpha$ and IL-6. TNF- $\alpha$ antagonist therapy restored the peripheral $\mathrm{V} \delta 2 \mathrm{~T}$ cell in RA.

Conclusions Elevated TNF- $\alpha$ in RA patients induced high expression of CCR5 and CXCR3 on V82T cells, which subsequently promote $\mathrm{V} \delta 2 \mathrm{~T}$ cells infiltrate into synovium and play an important role in the pathogenesis of RA. V $\delta 2 \mathrm{~T}$ cell is a promising potential biomarker and therapeutic target of RA.

\section{MORINGA OLEIFERA LAM AMELIORATES ADJUVANT INDUCED ARTHRITIS VIA INHIBITION OF INFLAMMATORY MEDIATORS AND DOWN-REGULATION OF MMP3 AND MMP-9 PROTEINS}

'P Pandey*, ${ }^{2} \mathrm{PC}$ Bhatt, ${ }^{1} \mathrm{~V}$ Kumar. ${ }^{1}$ Sam Higginbottom Institute of Agriculture - Technology and Sciences, Department of Pharmaceutical Sciences, Allahabad, India; ${ }^{2}$ JAMIA HAMDARD, pharmacognosy and phytochemistry, Delhi, India

\subsection{6/lupus-2017-000215.75}

Background and aims Rheumatoid arthritis (RA) is an autoimmune disease, which induces systematic and typical inflammation and affects $1 \%$ younger population Worldwide. Natural products are being preferred over the available treatment regimes for arthritis. Moringa oleifera has been very popular ethanomedicine for the treatment of inflammatory disorders in North-eastern part of India. With this background, the current investigation was carried out to scrutinise the anti-arthritic potential of Moringa oleifera in complete fruend's adjuvant (CFA) induced arthritis animal model.

Methods In the present investigation, we used the CFA, turpentine and formaldehyde into the sub-plantar region of hind paw of rats for induction of arthritis. After induction, joint diameter, arthritis score and body weight were estimated at regular interval. We studied the effect of plant extract on proinflammatory cytokines and inflammatory mediator, respectively. Histological architecture and other changes were also studied.
Results Oral treatment of $M O$ at doses of 25, 50 and $100 \mathrm{mg} / \mathrm{kg}$ significantly $(\mathrm{p}<0.001)$ down-regulated joint inflammation as evidenced via reduction in the joint diameter, arthritic score and inflammatory cell infiltration.

Conclusions MO treatment were found to reduce pro-inflammatory cytokines (TNF- $\alpha$, IL- 6 and IL-1 $\beta$ ) and inflammatory mediators $\mathrm{PGE}_{2}$ and $\mathrm{COX}-2$ in a dose dependent manner. $\mathrm{MO}$ also down-regulated the NF-kB in adjuvant immunised joint. Apart from these findings $M O$ abrogated degrading enzymes, which was evident from down-regulated protein expression of MMP-3 and MMP-9. Our findings clearly indicate the anti-arthritic potential of $M O$ via inhibition of NF-kB pathway.

\section{RASGRP4 EXPRESSION IN RHEUMATOID SYNOVIUM PLAYS A CRITICAL ROLE VIA RAS- MAPK PATHWAY}

S Shimamura*, S Yasuda, Y Shimizu, M Kono, M Kato, K Oku, T Bohgaki, T Atsumi. Hokkaido University Graduate School of Medicine, Division of Rheumatology- Endocrinology and Nephrology, Sapporo, Japan

\subsection{6/lupus-2017-000215.76}

Background and aims Ras activation as well as MAP kinase (MAPK) phosphorylation is known in the synovial tissues from patients with rheumatoid arthritis (RA). RasGRP4 is a guanine nucleotide exchange factor for small GTPase Ras and is expressed predominantly in the mast cells, monocytes and neutrophils. We previously identified ectopic expression of RasGRP4 in fibroblast-like synoviocytes (FLS) of a subset of RA patients, inducing proliferation of FLS (Kono $\mathrm{M}$ et al. Arthritis Rheumatol. 2015). Farnesyltransferase inhibitors (FTIs), prevent farnesylation of Ras, are known to prevent human tumour cell proliferation but the effect for proliferation of FLS is still unknown.

The aim of this study is to clarify the molecular mechanisms of how RasGRP4 induces proliferation of FLS and evaluate the effect of FTI on the proliferation of FLS.

Methods FLS or HEK293 cells were transfected with expression vector that encodes hRasGRP4. Phosphorylation of Raf, MEK, Erk, JNK and p38MAPK was evaluated in transfected cells using Western blotting. FLS were treated with tipifarnib, one of FTIs and cell proliferation was evaluated using BrdU Assay.

Results In HEK293 cells forced to express RasGRP4, RafMEK-Erk pathway as well as p38MAPK was readily phosphorylated at their steady state. FLS decreased RasGRP4 expression during multiple passages. RasGRP4 transfection into such cells recovered MAPK phosphorylations, especially of Erk and p38 MAPK. FLS treated with tipifarnib downregulated their proliferation.

Conclusions RasGRP4 expression in FLS from RA patients contributes to the activation of Erk and p38MAPK signalling pathway. The inhibition of FLS proliferation by FTI was suggested as an alternative treatment in RA. 